\title{
Téoros
}

Revue de recherche en tourisme

\section{Les parcs nationaux du Québec : Forillon, l'Archipel de Mingan et la Mauricie}

\author{
Solange Dion, Daniel Gosselin et Luc Perron
}

Volume 14, numéro 1, printemps 1995

Les parcs : des réseaux en mutation

URI : https://id.erudit.org/iderudit/1077055ar

DOI : https://doi.org/10.7202/1077055ar

Aller au sommaire du numéro

Éditeur(s)

Université du Québec à Montréal

ISSN

0712-8657 (imprimé)

1923-2705 (numérique)

Découvrir la revue

Citer cet article

Dion, S., Gosselin, D. \& Perron, L. (1995). Les parcs nationaux du Québec :

Forillon, l'Archipel de Mingan et la Mauricie. Téoros, 14(1), 37-41.

https://doi.org/10.7202/1077055ar d'utilisation que vous pouvez consulter en ligne. 


\title{
Les parcs nationaux du Québec : Forillon, I'Archipel de Mingan et la Mauricie
}

\author{
Solange Dion, Daniel Gosselin et Luc Perron
}

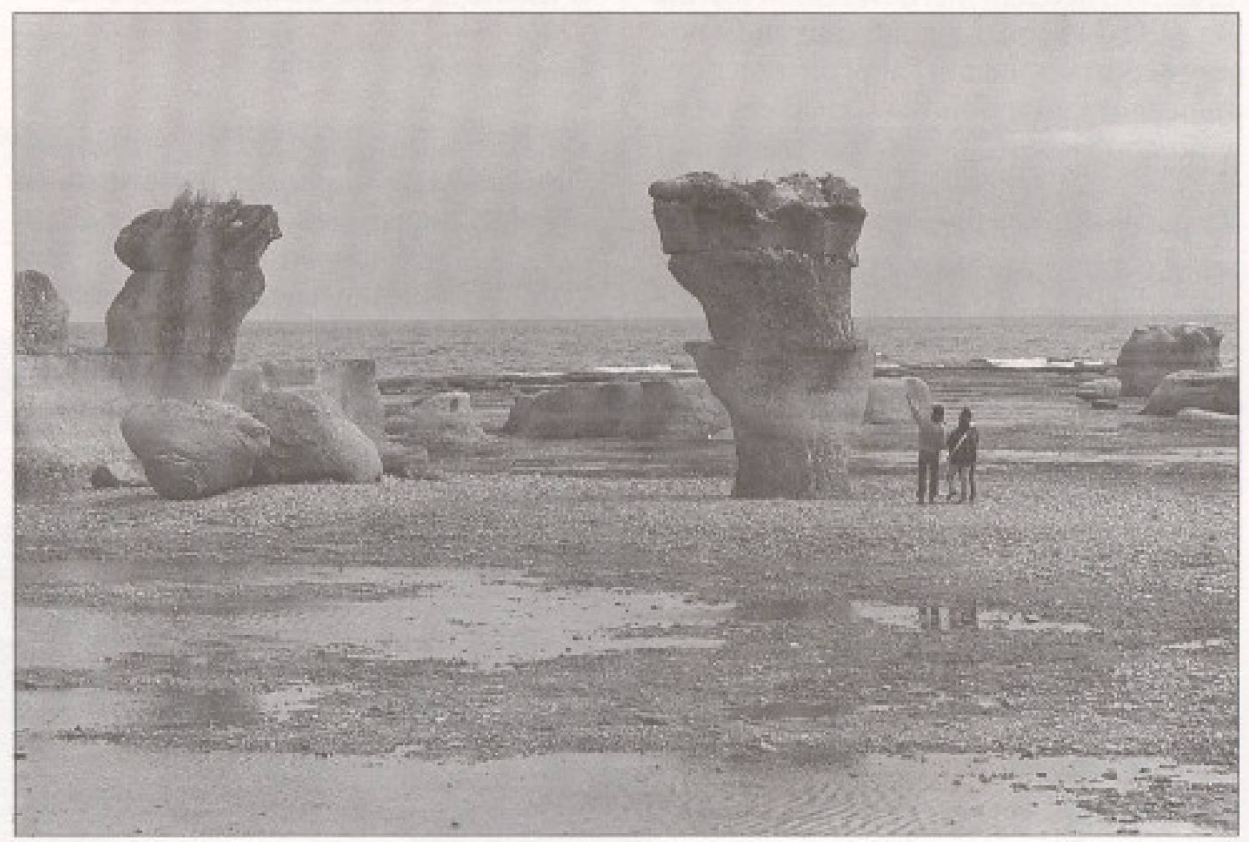

Réserve de parc national de l'Archipel-de-Mingan. (Photo: Jacques Bardsell, Pares Canada)

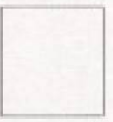

Leréseau desparcsnationauxdu Canada veut représenter les diverses régions naturelles du territoire canadien. Il a été créé dans le but de protéger et de conserver des sites exceptionnels de notre patrimoine naturel pour le bénéfice des générations actuelles et futures de Canadiens et de Canadiennes. Au Québec, le parc national Forillon, la réserve de parc national de l'Archipel-de-Mingan et le parc national de la Mauricie sont les trois sites sous la responsabilité de Parcs Canada.

Au fil des ans, les parcs nationaux sont devenus des pôles d'attraction importants pour la pratique d'activités de loisirs et de plein air. La qualité des aménagements et des services ainsi que l'offre d'activités de loisir et d'interprétation permettent aux visiteurs des pares de vivre des expériences de plein air dans un milieu naturel dont l'intégritéćcologique est assurée. Destinations touristiques en soi, les pares nationaux du Québec joueront un rôle de plusen plusimportantdansl'organisation del'offre récréo-touristique de leur région d'appar- tenance et, de façon plus globale, du Québec.

Cet article veut présenter les trois parcs nationaux du Québec et différents aspects del'utilisation qui en est faite actuellement par le public. Ainsi, autant pour Forillon, pour l'Archipel de Mingan que pour la Mauricie, la fréquentation des sites et le profil des clientèles actuelles seront présentés. Dans le cas du parc national de la Mauricie et de la réserve de parc national de l'Archipel-de-Mingan, les résultats des plus récentes études sur l'impact économique deces sites seront aussi transmis a fin de démontrer l'importance de leur contribution à l'économie régionale. Enfin, nous aborderons les perspectives d'avenir des trois pares nationaux du Québec.

\section{Le parc national Forillon}

Leparcnational Forillon est situéà l'extrémité nord-est de la péninsule gaspésienne, à quelque 700 kilomètres de Québec. Le parc fut créé en 1970 aux fins de préserver un exemple représentatif de la région na- 
turelledesmonts Notre-Dame et Mégantic, où se dressent les plus hauts massifs montagneux de la partie habitée de l'est du Canada. Sous le thème de l'harmonie entrel'Homme, la Terreet la Mer, Forillon a pour mission d'assurer la sauvegarde à perpétuité de ce territoire de $240 \mathrm{~km}$ carrés et de certains élếments de la région marine du golfe du Saint-Laurent, tout en favorisant la connaissance et l'appréciation de ce milieu par l'ensemble des Canadiens et les visiteurs d'aujourd'hui et de demain.

Depuissa création, le parcnational Forillon a fait l'objet de plusieurs phases de développement qui se sont traduits en une offre diversifiée d'aménagements et de services. Ceux-ci sont pour la plupart répartis à l'intérieur de trois secteurs d'utilisation où sont regroupés les principaux centres d'activités, les terrains de camping et les sites récréatifs ou d'animation sur la thématique du parc. Les toponymes locaux des lieux de rassemblement évoquent des facettes significatives de l'histoire de Forillon : Penouille, Fort-Péninsule, CapBon-Ami, Petit-Gaspé, Grande-Grave, Anse-Blanchette, Anse-aux-Sauvages, CapGaspé, etc.

\section{La fréquentation}

En comparaison avec la décennie précédente, Forillon a connu au début des années 1990 une importante augmentation de sa fréquentation et de son utilisation, résultat concret de campagnes de publicité menées à la fin des années 1980. Exprimée en termes de evisites pour apprécier le patrimoine naturel et culturel ou pour se recréer», la fréquentation du parc a atteint des sommets depuis sa création, dépassant les 200000 visites en 1989.

Subissant l'influence de multiples facteurs socio-économiques au début des années 1990, la fréquentation du parc, quoique toujours très intense durant le mois de juillet et au début du mois d'août, a néanmoins connu un certain fléchissement pour se rétablir autour de 1700000 visites en 1993. Quelque $77 \%$ des visites dela saison estivale ont lieu en haute saison (juillet et août).

Alors que l'on estime la fréquentation des secteurs etaires d'activités à 135000 visites différentes en 1993, le nombre de joursvisite s'accroît à environ 194000 lorsque l'on tient compte des séjours de tous les campeurs dans le parc. La clientèle des compeurs a atteint des sommets au tournant de la décennie pour dépasser 59000 nuitscampeur ou 20200 nuits-site de camping. En 1993, les trois terrains de camping du parc (333 emplacements) ont connu des taux d'occupation variant de $67 \%$ à $73 \%$ jusqu’à la fête du Travail; il est à noter que le mois de juillet accapare à lui seul près de la moitié de toutes les nuits-campeur.

\section{La clientèle}

Les visiteurs du parc national Forillon proviennent pour la plupart des principaux marchés touristiques québécois et étrangers; la région immédiate contribue pour $20 \%$ des visites en haute saison, le reste du marché québécoispour $58 \%$ etles marchés de l'extérieur du Québee pour $22 \%$; la clientèle en provenance de la France, en hausse actuellement en raison de promotions particulièrement ciblées, comptait pour $7 \%$ des visites enregistrées au parc au début des années 1990. Environ $7 \%$ des visites sont attribuables aux Américains. En bassesaison, la fréquentation actuellese répartit différemment, affichant une plus grande proportion de résidents de la municipalité de Gaspé, et une majorité d'Européens, des Français surtout.

La grande majorité des visiteurs se présentent au parc pour y passer une journée seulement, souvent pas plus de quelques heures. Le quart y séjournent en camping dans le parc $(16 \%)$ ou dans des établissements ou terrains de comping de la région (10 \%). En regard des besoins d'information et d'accompagnement qui sont différenciés selon le type de visite au parc, nous Les visiteurs du parc se présententen groupes d'adultes principalement mème si une bonne proportion des groupes $(25 \%)$ comptent aussi deux enfants/adolescents ou plus. Ils recherchent des expériences de séjour en nature de grande qualité comportant des dimensions éducative et sécuritaire assurées dans un paysage grandiose.

\section{La réserve de parc national de l'Archipel-de-Mingan}

La réserve de parc national de l'Archipelde-Mingan se situe en Moyenne CôteNord le long du fleuve Saint-Laurent, au nord de l'ìle d'Anticosti. Cette réserve représente l'une des trois sous-régions des Basses-TerresduSaint-Laurentquis'étend de la pointe sud de l'Ontario et du Québec jusqu'au sud du Labrador. La réserve de parc national (RPN) de l'Archipel-deMingan regroupe neuf cents îles et îlots, dont une quarantaine de surface plus importante, sur une distance de plus de 175 km qui ont été mis de côté jusqu'au règlement des revendications territoriales des Attikameks-Montagnais. La réserve comporte des éléments naturels uniques tels les monolithes aux formes parfois énigmatiques sculptés par le travail érosif delamer. Desmacareuxet d'autresoiseaux de mer nichent sur certaines de ces îles du golfe Saint-Laurent tandis que des marsouins, des phoques et des baleines se nourrissent dans les eaux riches du large.

Les aménagements et services offerts par Parcs Canada dans le parc et sa région environnante sont limités et resteront modestes afin de préserver le caractère naturel particulier de cetarchipel. La RPN de l'Archipel-de-Mingan créée en 1983 poursuit sa phase de développement à un rythme façonné par la croissance de sa fréquentation tout en protégeant le caractère d'isolement et la fragilité du milieu naturel de l'archipel. Les aménagements sur la terre ferme refléteront, une fois complétés, la vocation distincte assignée aux trois pôles d'accueil: à Longue-Pointe, le monde marin, à Mingan, l'histoire et les traditions associées à la culture amérindienne et à Havre-Saint-Pierre, le monde insulaire.

\section{La fréquentation}

La RPNde l'Archipel-de-Mingan a connu une relative stabilité de sa fréquentation en 1993. Cette situation permet au pare d'enregistrer une fréquentation se situant depuis 1990 entre 25000 et 27000 visites annuellement. Ce niveau de fréquentation secompareavantageusementaux $10500 \mathrm{et}$ 21000 visites dénombrées en 1984 et 1986 respectivement. Au cours de cette période, la fréquentation de la RPN a pu bénéficier des effets positifs relatifs à la publicité entourant l'acquisition des îles par le gouvernement fédéral et del'image de prestige associée à l'établissement d'un parc national. Après avoir atteint des sommets en 1990, la fréquentation semble fortement sensible aux cycles économiques de l'́́conomie provinciale. En dépit de ces fluctuations, la fréquentation demeure extrêmement concentrée durant la période estivale et atteint des sommets durant la deuxième quinzaine de juillet, période qui coincide avec les vacances des travailleurs de la construction et des secteurs affiliés au 


\section{Segments - Haute saison}

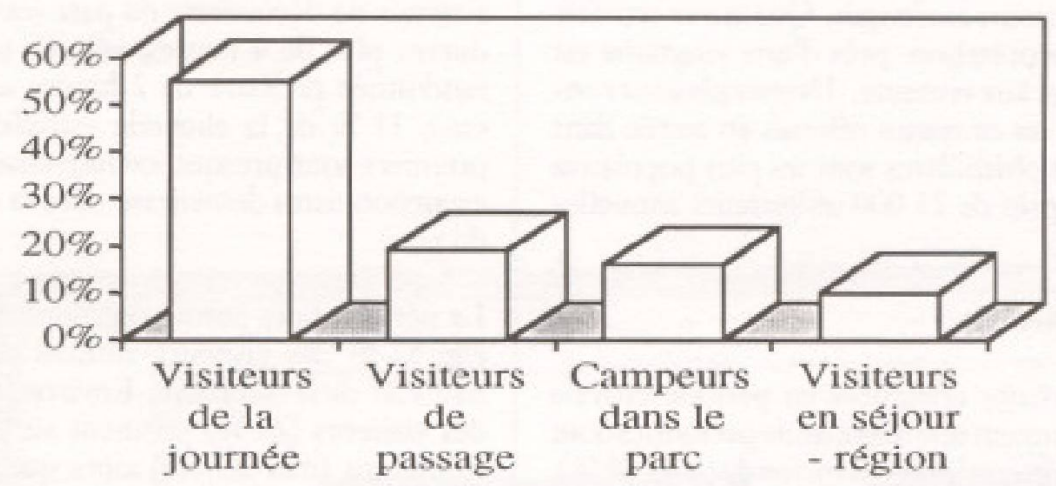

Québec. Enfin, plus de 25000 personnes ont utilisé les centres d'accueil et d'interprétation situés à Havre-Saint-Pierre et à Longue-Pointe.

\section{La clientềle}

Les résultats des enquêtes menées auprès des touristes et deshabitantsdela Minganie en 1989 et d'analyses approfondies permettent de décrire le profil type de la clientèle du parc. La clientèle touristique contribue pour $82 \%$ de la fréquentation du parcalors quela clientèle provenant des cinq municipalités associées directement à la Minganie(Havre-Saint-Pierre, LonguePointe, Mingan, Baie-Johan-Beetz et Aguanish) le fait pour $18 \%$.

La clientèle touristique est composée essentiellement de Québécois (94\%) dont près de la moitié proviennent de la région administrative de Montréal. Plus de $90 \%$ des visites sont effectuées lors des mois de juillet et août. Près de $80 \%$ des visiteurs touristes ont indiqué que la présence de la RPNà Mingan était la seule raison $(20 \%)$ ou une des raisons $(57 \%)$ expliquant leur choix de la Cote-Nord comme destination devacances, La très grande majorité de ces groupes sont composés d'adultes seulement. Les visiteurs touristes dont la visite nécessite au moins un coucher passent en moyenne 10,6 nuits lors de leur déplacement et séjournent en Minganie en moyenne 3,4 nuits. Selon les résultats de l'enquête de 1989, les groupes de visiteurs touristes dépensaient en moyenne $830 \mathrm{~S}$ pour l'ensemble de leur voyage dont $43 \%$ l'était en Minganie, soit 350 Sen moyenne par groupe de visiteurs touristes. Cette clientèle a utilisél'hôtel ou fait du camping durant son voyage dans des proportions similaires (35\%). La dientèle touristique accédant principalement aux îles par le biais d'une croisière y passe environ deux heures à observer la nature $(26 \%)$ et a faire une randonnée pédestre $(24 \%)$; toutefois, près de $37 \%$ des touristes (approximativement 10000 personnes) en Minganie quittent la région sans avoir pu visiter le parc, les îles proprement dites, reflétant ainsi l'appréciation des services exprimée à l'égard des tarifs de croisière et des transporteurs privés.

La clientèle régionale est composée des groupes de visiteurs provenant de HavreSaint-Pierre dans une proportion de plus de $80 \%$. Ils accèdent au parc principalement durant les mois de juin et de juillet avec une embarcation privée $(71 \%)$ et passent deux fois plus de temps sur les îles que les touristes.

\section{Les retombées économiques}

Les retombées économiques représentent les effers (gains supplémentaires) attribuables aux efforts déployés par Parcs Canada pour protéger des éléments représentatifs de notre patrimoine naturel sur les tierces parties comme les employés locaux, les fournisseurs et les habitants. L'évaluation prend en considération les revenus et les emplois soutenus dans les municipalités sises entre Sept-Iles et BaieJohan-Beetz.

Les retombées économiquesontété mesurées à l'échelle du territoire de la Moyenne Côte-Nord pour les dépenses de Parcs
Canada et à celle de la Minganie pour les dépenses aturibuables aux touristes dans le cadre de la préparation du plan de gestion du parc, approuvé en juin 1992. Cette évaluation s'est particulièrement concentrée sur la contribution du parc d'ici 1999 quant à ses perspectives de développement et en fournissant des ordres de grandeur quant aux retombées provenant des dépenses d'investissement envisagées, des dépenses d'exploitation et d'une fréquentation anticipéeà près de 30000 visites dans un avenir rapproché. L'impact économique lié aux dépenses d'investissement passées et projetées est beaucoup moins élevê que les autres sources d'impact puisque celles-ci sont sujettes à des fuites importantes directement vers d'autres régions du Québec étant donné le nombre restreint d'entreprises spécialisées et la difficulté de répondre aux besoins particuliers de Parcs Canada,

Les dépenses touristiques évaluées à près de 3 millions (M) $\$$ annuellement, en fonction d'un niveau de fréquentation de 30000 visites, procureront directementaux résidents de la région des possibilités de revenus de 355000 Set foumirontdu travail équivalant à 14,2 années-personnes, les secteurs de la restauration et de l'hébergement étant les principaux bénéficiaires de ces répercussions positives. Ces dépenses auront un effet d'entrainement possiblement fort limité sur l'ensemble de l'économie régionale considérant sa strucrure économique moins diversifiée. Les effets indirects et induits représentent près de $10 \%$ des effets directs estimés. Cette évaluation nous fournit une bonne indication de l'apport annuel relatif des dépenses attribuables aux touristes (prèsdu tiers des revenus directs proviennent des dépenses d'exploitation de parc).

L'importance de la contribution économique de Parcs Canada en Minganie tout particulièrement, les dépenses récurrentes d'exploitation du parcetcelles des touristes provenant de l'extérieur de la région de la Moyenne Côte-Nord, est liće au fait que ces dépenses sont effectuées dans une région située en périphérie des grands centres urbains québécois. Ces sommes injectées annuellement dans l'économie régionale constituent une source stable de revenus et d'emplois (près de 1,5 M S en revenus totaux et plus de 50 années-personnes de travail dont près de $60 \%$ sont attribuables a l'embauche directe de Parcs Canada) etcontribuent à unediversification 
del'économie régionale (l'industrietouristique: secteurs de la restauration et de l'hébergement).

\section{Le parc national de la Mauricie}

La région naturelle précambrienne du Saint-Laurent et des Grands-Lacs est représentée au sein du réseau des parcs nationaux par le parc national de la Mauricie. Domaine de lacs et de forêts où vit une faune diversifiée, le parc national de la Mauricie (PNLM) présente le riche paysage du Bouclier canadien sous le thème de l'aHERITAGE LAURENTIENm. Bénéficiant d'une localisation facilement accessibleaux principauxmarchésduQuébec, puisqu'il est situé à peine à $70 \mathrm{~km}$ au nord de Trois-Rivières, le parc national de la Mauricie accueille annuellement près de 250000 visiteurs. Les 544 kilomètres carrés d'aires protégées et conservées disposent de plusieurs aménagements etservices.

\section{La fréquentation}

Depuis sa création en 1970, le PNLM a atteint un plateau quant au nombre de visites annuelles qui ysont effectuées. Avec environ 250000 visites, le parc maintient une vitesse de croisière depuis quelques années. On constate cependant que près de la moitié des visites annuelles ( $49 \%$ ) sont concentrées dans les mois de juillet et d'août. Certaines zones aménagées du parc atteignent, durant cette période, les limites de leur capacité d'accueil. Ce phénomène entraîne la nécessitéd'en restreindre régulièrement l'achalandage.

D'autre part, à partir de la fin de semaine précédant la Saint-Jean-Baptiste jusqu'à la fête du Travail, les taux d'occupation des trois terrains de camping du parc sont très élevés. Ainsi, les 208 emplacements du camping de Rivière-à-la-Pêccheont un taux d'occupation de $70 \%$, le camping Wapizagonke, avec ses 219 emplacements, est occupé dans une proportion de $89 \%$, alors que les 90 emplacements du camping Mistaganceatteignentun taux d'occupation de $82 \%$. Le nombre total de nuits-site louées tourne annuellement autour de 35000 . Notons que les campeurs représentent le tiers $(33 \%)$ de la fréquentation en période estivale. D'ailleurs, lorsqu'on prend en considération les séjours descampeurs et des canots-campeurs, la fréquentation du PNLM atteint près de 400000 jours-visite, ce qui en fait le parc national le plus fréquenté au Québec. Les Services d'accueil du parc permettent à plus de 130000 utilisateurs d'être informés sur les activités disponibles et d'être orientés vers les secteurs aménagés. Quant aux activités d'interprétation, près d'une vingtaine est offerte aux visiteurs. Depuis plusieurs années, les causeries offertes en soirée dans les amphithéâtres sont les plus populaires avec près de 25000 utilisateurs annuellement.

\section{La clientèle}

Les visites effectuées en période estivale regroupentunemajoritédepersonnes dont l'origineestla région mêmedu parc $(54 \%)$, soit la Mauricie/Bois-Francs (04). L'ile de Montréal (06) fournit $10 \%$ de la clientèle du parc en été alors que, près du tiers $(32 \%)$, est issue des autres régions du Québec. Les visiteurs de l'extérieur du Québec forment, quant à eux, $4 \%$ de la clientèle estivale du parc. Cette répartition amène à constater que, depuis 10 ans, la part des visiteurs régionaux, bien que demeurant majoritaire, est de moinsen moins importante par rapport au total de la fréquentation en été au PNLM. Ainsi, elleest passée de $75 \%$ en 1981 à $54 \%$ en 1991 . Cette situation représente une augmentation du rayon d'attraction de ce parc. Ce phénomène s'est surtout opéré vers les marchés à l'ouest du parc, soit la région de Montréal (06) et ses régions périphériques (Laval, Lanaudière, Laurentides, Montérégie).

Bien qu'une majorité ( $55 \%$ ) de visiteurs soient des excursionnistes (effectuant un aller-retour de leur domicilevers le parcen une journée), une portion importante de la clientèle estivale provient du marché touristique $(45 \%)$. Le parc national de la Mauricie est sans contredit un facteur déclencheur de déplacement récréo-touristique puisque près de neuf visiteurs sur dix $(88 \%)$ en ont fait la destination principale de leur déplacement ou voyage. C'est surtout à cause de la beauté naturelle des paysages et de la proximité du site que le PNLM est choisi comme destination.

Les clients du parc national de la Mauricie sont de fidèles utilisateurs, 4,4 visites en moyenne par été, et des habitués du parc puisque seulement $26 \%$ des visiteurs en sont à une première visite. En période de pointe, la clientèle du parc national de la Mauricie se divise en deux groupes selon l'expérience de séjour vécue au parc. Ainsi, les visiteurs qui effectuent des activités de type récréa tif(baignade, balade en voiture, pique-nique, etc.) représentent $69 \%$ de la clientèle et les visiteurs qui pratiquent des activités de découverte du parc (canotage durant plus de 4 heures, vélo en sentier, randonnée pédestre de 2 heures et plus, etc.), $31 \%$ de la clientèle estivale. Les premiers sont presque exclusivement des excursionnistes demeurant dans la région du parc.

La période hors pointe regroupe quant à elle $51 \%$ des visiteurs annuels du parc national de la Mauricie. Environ le quart des visiteurs $(24 \%)$ viennent au parc au printemps (mai et juin) alors que la fréquentation en automne (septembre=octobre) compte pour $16 \%$ des visites effectuées et que les skieurs de randonnée, en hiver (novembre à avril), forment $11 \%$ de la fréquentation annuelle. Au cours de l'ensemble de la période hors pointe, les visiteurs régionaux représentent plus de $70 \%$ de la fréquentation. Il s'agit principalement de visiteurs excursionnistes qui sont des habitués du parc. Regrou= pantsurtoutdesadultes $(80 \%)$, lesvisiteurs de la période hors pointe fréquentent le parc pour des activités particulières variant selon les saisons. Ainsi, la pêche estl'activité principale au printemps, alors que l'exploration des sentiers, l'observation de la faune et des couleurs de la forêt prédominent à l'automne. En hiver, le ski de randonnée est l'activité pratiquée par la quasi-totalité des visiteurs.

\section{Les retombées économiques}

Du point de vue de l'impact économique, le parc national de la Mauricie joue un rôle significatif dans la région touristique du Coeur-du-Québec. Positionné comme un élément d'attraction majeur dans son milieu, il amène des retombées secondaires à partir de trois sources principales. La première est liée à l'existence du parc et aux argents nécessaires à son fonctionnement et son entretien. La seconde source est issuedes projets d'investissements que Parcs Canada y met en oeuvre. Enfin, une troisième source d'impact économique met en cause les dépenses des visiteurs lors de leur passage dans la région et, spécifiquement, celles attribuables à leurs séjours au parc. L'utilisation d'un modèle d'impact économique a permis d'estimer les retombées totales (directes, indirectes et induites) attribuables à la présence du parc national dela Mauriciepour la région du Coeur-du- 
Québec. En 1992-1993, l'administration du parc national de la Mauricie a dépensé 3,6 millions (M) \$ (entretien, fonctionnement et investissements), dont les deux tiers (2,54 M S) ont été déboursés dans les communautés environnantes du parc (incluant les salaires versés aux employés du parc) et a créé 140 emplois. Les dépenses de Parcs Canada dans la région ont en plus entraîné des retombées économiques indirectesetinduites sur l'économierégionale de l'ordre de 2,7 M S en revenus de travail et d'environ 117 années-personnes, soit l'équivalent d'environ 350 emplois sai= sonniers ou non. On note par ailleurs, au plan des retombées économiques dues à la présence du parc dans la région de la Mauricie que:

- en 1993, les visiteurs ont dépensé plus de $6,0 \mathrm{M}$ \$dansla région mauricienne;

- les campeurs représentent $13 \%$ des visites-personne et sont directement responsables de près de $40 \%$ des dépenses totales des visiteurs attribuablesauparcnationaldela Mauricie;

- les visiteurs régionaux représentent $57 \%$ des visites-personne et ne contribuent que pour $34 \%$ de ces dépenses;

- il faut attirer un campeur de l'extérieur de la région contre cing visiteurs diurnes de la région pour générer les mêmes niveaux de dépenses et d'impact économique;

- plus de $2,8 \mathrm{M}$ S ont été versés par les visiteurs aux habitants de la région sous forme derevenus de travail; leurs dépenses supportent plus de 350 emplois saisonniers dans la région.

\section{L'avenir des parcs nationaux}

On s'entend généralement pour reconnaître les pares nationaux comme des produits touristiques de qualité exceptionnelle en raison de l'unicité de leurs paysages et de la qualité de leurs infrastructures. Ces lieux privilégiés de conservation, d'éducation et de récréation se présentent comme des destinations récréotouristiquesd'intérêt et sont appelésà jouer un rôle de plus en plus important en raison, notamment, des retombées sociales et économiques qu'ils peuvent engendrer. Forillon

A cet égard, le pare national Forillon est devenu au fildes ans une destination pour un nombre accru de touristes qui font le cir- cuit de la Gaspésie. Sans encore menacer la destination traditionnelle du wtour de la Gaspésies que constitue Percé, Forillon n'en a pas moins acquis la réputation d'une étape incontournable chez les vacanciers de la péninsule gaspésienne.

Il est à prévoir que le parc national Forillon continuera d'attirer un grand nombre de touristes en Gaspésie; les amateurs d'écotourisme y afflueront en raison de sa haute valeur écologique alors que les familles et les autres touristes gaspésiens y trouveront des aménagements qui répondent à leurs besoins de communion avec des valeurs patrimoniales «Nature - Cultures généreusement partagées. Le caractère commémoratif de la vie du pêcheur de la Gaspésie, abondamment illustré par les scénographies présentées à GrandeGrave, tout comme l'accessibilité des sites d'observation de la nature, de la faune en particulier, conferent à Forillon des atouts majeurs pour une mise en marché internationale,

La stratégie marketing privilégiée favorisera la concertation de tous les intervenants du tourisme en Gaspésie (association touristique régionale (ATR), autres ordres de gouvernement et organismes privés) afin d'en maximiser les retombées et d'harmoniser une offrede service ca ractérisée par la qualité exceptionnelle d'expérience de séjour qu'elle permettra. A cette fin, en raison de la capacité d'accueil du parc et de celle de la région, l'allongement de ce qui est considéré comme étant la haute saison sera l'objectif premier en matière de fréquentation au cours des prochaines annếes.

\section{L'Archipel de Mingan}

Lesactionsenvisagées par Parcs Canada en ce qui a trait à la réserve de parc national de l'Archipel-de-Mingan permettront minimalement de maintenir les taux de fréquentation actuels du parc et les retombées découlant de ce niveau d'activité. Les communaurés situées dans la région immédiate du parc seront appelées à jouer un rôle prépondérant afin de réaliser le plein potentid touristique de cette attraction. Le caractère exceptionnel du site et le mandat de protection de l'intégrité écologiquede Pares Canada permetra d'assurer la pérennité de ce site.

\section{La Mauricie}

Le développement du parc national de la Mauricietel que prévu au plan directeur est à toute fin pratique complété. Le parc fournit ainsi aux visiteurs une expérience privilégiée dans un cadre naturel exceptionnel où il est facile d'en apprécier les ressources. Toutefois, on noteau parc, lors des périodes de pointe, un achalandage dépassant la capacité d'accueil actuelle du milieu naturel et de ses équipements ce qui ne correspond pas au type d'expérience que Parcs Canada veut offrir aux visiteurs à l'intérieur de ce territoire. Malgré cet achalandage, peu de développements reliés au produit touristique ont vu le jour en périphérie depuis 25 ans.

Ces faits étant établis, les interventions à venir devront mettre en priorité une vision intégrée des actions régionales en vue d'accroitre les retombées sociales et économiques de la présence du parc national dans cette région. Les actions devront aussi supporter le rehaussement du profil des equipements et des infrastructures localisés dans le parc et dans sa périphérie tout en contribuant à l'atteinte de nos objectifs de diversification des clientèles tout au long de l'année. $f$

\section{REFEERENCES}

L'ECART-TYPE, Étude auprès des visiteurs de la ré ser vo de parc nationaldel'Archipel-de Mingan. rapport final, mars 1990.

L'ÉCART-TYPE, Etude auprès des visiteurs du parc national Forillon, 1990-1991, mars 1992

PAACS CANADA, L'avenir du parc, Révision du plan directeur, parc national Forillon. Patrimoine cafadien.

PARCS CANADA, Plan de gestion: Reserve de parc national de I'Archipel-de-Mingan, Québec, Service de la planitication, juin 1992.

PARCS CANADA, Plan de gestion revisé du parc national de la Mauriclé, Québec, Service de la planification, avril 1992.

PAACS CANADA, Plan de sorvices du parc national de la Mauricie, aoùt 1991

PAFCS CANADA. Rapport statistique 1993, région du Outhes. Patrimgine canadi en.

PARCS CANADA, Foisy, LUC. Les loisirs at la préservation des parcs nationaux canadiens, Frutentation dans le cadre du collocue international sur le loisir, Université du Québeca Trois-Fivieres, novembre 1994

PARCS CANADA ET L'ECART-TYPE, Etude sur los clienteles actuelles du parc national de la Mauricie, mars 1992

ZINS BEAUCHESNE ET ASSOCIES, Etude de marchó pour le pare national Forillon, janvier 1993. 